\title{
Contribution des traceurs naturels à l'évaluation des ressources en eau souterraine
}

PAR

\author{
G. Castany, A. Marce et J. Margat \\ Service Géologique National, Orléans
}

\section{Introduction}

Pour apprécier clairement comment les données fournies par l'observation des traceurs naturels, et tout particulièrement par les isotopes du milieu, peuvent servir en pratique à l'évaluation des ressources en eau souterraine, un préalable est nécessaire : bien définir la conception actuelle des ressources en eau souterraine (*).

On s'est longtemps contenté d'assimiler ces ressources au débit naturel global des nappes, équivalant à assez long terme à leur alimentation, les associant ainsi au bilan et à l'idée d'un équilibre à sauvegarder sous peine de "surexploitation".

Mais cette conception purement physique, "hydrologique", des ressources en eau souterraine conduit à définir uniquement des ressources "potentielles" ou naturelles dont l'utilité pratique est très restreinte. De plus, elles ont pour défaut de rester globales pour un réservoir aquifère donné, et d'éliminer l'intérêt d'utiliser également, dans certaines conditions, les ressources non renouvelables.

Les ressources exploitables effectives sont très généralement inférieures aux ressources naturelles, par l'effet de diverses contraintes physiques ou économiques qui limitent les possibilités d'exploitation. Mais, dans certains cas, au contraire, ces ressources peuvent dépasser largement les ressources naturelles (beaucoup d'aquifères alluviaux très liés aux rivières). De même, les quantités d'eau prélevables pendant une longue

(*) : Dans ces définitions, nous n'envisageons que les ressources quantitatives en laissant de côté les aspects qualitatifs des ressources (et des demandes). Ceux-ci n'en sont pas pour autant indépendants et doivent évidemment être pris en compte dans une définition complète des ressources en eau, ay ant une valeur pratique. durée dans les grands aquifères captifs peuvent être très supérieures à leurs ressources renouvelables naturelles.

L'évaluation des ressources en eau souterraine n'est pas en fait indépendante de la demande d'eau à satisfaire (localisation, variabilité des quantités voulues, flexibilité, valeur d'usage, sans parler des exigences de qualité). C'est un problème sans solution unique. Elle revient à confronter un modèle d'offre à une gamme de "modèles de demande" et à opérer un choix plus ou moins optimal.

L'évaluation des ressources en eau souterraine - laquelle, en toute rigueur, n'a de sens que dans le cadre spatial d'un système aquifère bien délimité - suit très schématiquement la procédure suivante :

$1 /$ définition du système aquifère (géométrie, caractéristiques internes, conditions aux limites, dynamique).

2/ définition des contraintes (absolues ou plus ou moins flexibles) dont le respect peut être imposé :

contraintes physiques (alimentation du système, débits ou niveaux à conserver, qualité à maintenir), contraintes économiques (coût de production maximal fixé)

3/ élaboration de programmes d'exploitation (dispositif, régime) correspondant à différents scénarios de besoins en eau (exprimés ou imaginés)

4/ détermination des effets sur le système des programmes projetés,

5/ comparaison de ces effets et des contraintes,

6/ évaluation de la ressource exploitable c'est-à-dire choix du (ou des) programme(s) qui maximise(nt) (ou optimise(nt)) les prélèvements d'eau souterraine en respectant les contraintes (ce qui implique en général un certain nombre de "boucles" entre les stades 5 et 3$)\left(^{*}\right)$.

(*) N.B. - Cette ressource sera toujours révisable en cas de modification des contraintes ou/et de renouvellement du modèle de demande. 
Dans ce schéma, les informations apportées par les traceurs naturels vont essentiellement contribuer, parmi d'autres, à construire les modèles de système aquifère visés au stade 1 . L'utilité de ces informations pour l'évaluation des ressources sera donc fonction de leur contribution à l'élaboration de ces modèles.

La précision à rechercher pour décrire un système aquifère étudié dépend à la fois de sa complexité et du problème à résoudre. Mais, dans tous les cas, l'hydrogéologue a intérêt à réunir le maximum de données indépendantes - d'ordre géologique, géophysique, hydrologique et hydrodynamique, hydrochimique, etc. - dont seule la synthèse cohérente pemet de construire le schéma le mieux représentatif de la réalité. Chaque catégorie d'information fournit en effet un certain nombre de faits, mais laisse aussi généralement une marge d'incertitude entre diverses hypothèses. Le contrôle mutuel d'informations suffisamment indépendantes est le moyen le plus sûr, et souvent le plus économique, de les réduire.

Dans cette optique, les données apportées par l'étude des isotopes du milieu, et plus particulièrement le Carbone 14, le Carbone 13, le Tritium, l'Oxygène 18 et le Deuterium présentent le grand intérêt de fournir des informations entièrement indépendantes dont l'interprétation reliée aux autres données acquises par une étude hydrogéologique détaillée, peut contribuer efficacement à la connaissance :

- de la structure du système aquifère, notamment lorsqu'il s'agit de multi-couches du degré de liaison entre les différentes couches aquifères (drainances),

- des conditions aux limites du système,

- du mode d'alimentation et de renouvellement des réserves.

Cette contribution est particulièrement utile dans l'étude des grands systèmes aquifères captifs des bassins sédimentaires multicouches, pour laquelle les données acquises par les. méthodes d'investigations plus directes (forages, géophysique) sont toujours assez limitées pour des raisons essentiellement économiques.

Aussi est-ce surtout dans l'étude de systèmes de ce type que l'on a recours aux analyses des isotopes du milieu et à leur interprétation.
Parmi un certain nombre d'applications récentes, auxquelles il nous a été donné de participer, trois études, réalisées dans des conditions hydrogéologiques différentes, ont permis d'éprouver l'utilité des "techniques isotopiques" et de préciser les conditions générales de leur emploi, en insistant particulièrement sur l'utilisation du Carbone 14.

Nous ne ferons pas état ici d'une étude analogue réalisée antérieurement sur la nappe de l'Albien du Bassin de Paris par notre confrère $Y$. Vuillaume, dont les résultats, appuyés sur 60 points de mesure, ont déjà été publiés [4 et 5].

Il s'agit ici des études des nappes des Sables du "maestrichtien" et du Continental terminal du Sénégal (en collaboration avec l'A.I.E.A.), avec 80 points de mesure, de la nappe des calcaires miocènes de Cyrénäque en Lybie (réalisée dans le cadre du GEFLI) avec 19 points de mesure, et de la nappe des calcaires dolomitiques jurassiques d'AinBeni-Mathar au Maroc oriental (réalisée pour la Direction de l'hydraulique du Maroc) avec 10 points de mesure. Les résultats analytiques obtenus pour chacune de ces études ont été publiés en [6] par l'A.I.E.A. (1974), aussi n'a-t-il pas paru nécessaire de les reproduire ici.

\section{Aperçu hydrogéologique sur les systèmes aquifères étudiés}

L'aquifère profond du Sénégal, contenant la dite nappe des Sables "maestrichtiens", principale ressource en eau souterraine du pays, a fait l'objet d'études hydrogéologiques détaillées, précises $[1,2$ et 3$]$. Il est constitué principalement par une formation de sables et de grès tendres avec lits d'argile, d'âge maestrichtien, d'épaisseur relativement constante (moyenne de $200 \mathrm{~m}$ ) et de profondeur moyenne à $200 \mathrm{~m}$, couvrant la quasi-totalité du bassin sédimentaire, soit environ $150000 \mathrm{~km}^{2}$ (fig. 1). Les eaux souterraines, captives, dont la réserve totale est de l'ordre de $3.10^{12} \mathrm{~m}^{3}$, sont exploitées par une centaine de forages. Les zones d'alimentation directe actuelles sont localisées aux affleurements, en bordure du socle et à l'W dans la presqu'île du Cap Vert. Une carte

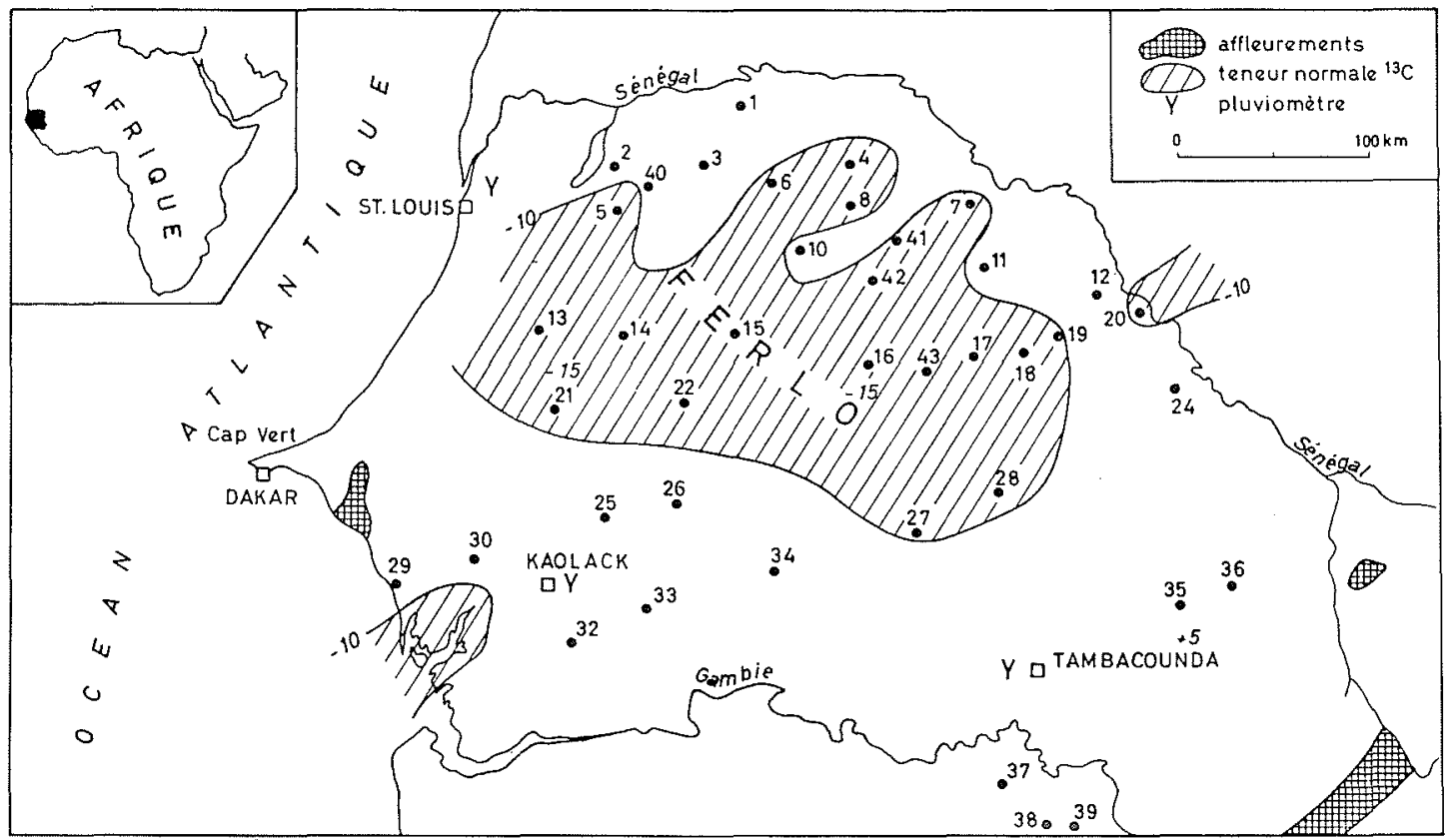

1, Nappe des sables "maestrichtiens" du Sénégal. Teneurs en ${ }^{13} \mathrm{C}$ 
piézométrique montre un écoulement général convergent des eaux souterraines du $S E$ vers le $N W$, avec deux gouttières centrales caractérisant une nappe à dépression centrale fermée. Le faible gradient hydraulique (1/10000 à 1/25000) traduit une très faible vitesse de filtration, avec une alimentation préférentielle par les affleurements du $S E$ et un confinement de l'écoulement sur le littoral. Des pompages d'essais ont permis de déterminer la transmissivité $\left(1.10^{-2}\right.$ à $2.10^{-4} \mathrm{~m}^{2} / \mathrm{s}$ ) et le coefficient de perméabilité moyen de $1.10^{-4} \mathrm{~m} / \mathrm{s}$. La nappe des Sables "maestrichtiens" est surmontée, au-dessus de formations de marnes et d'argiles constituant un toit semi-perméable (Paléocène + Eocène inférieur), par la formation argilo-gréseuse du Continental terminal, d'épaisseur variable (quelques dizaines à $150 \mathrm{~m}$ ) renfermant une nappe libre exploitée au $S$ et à l'W sur le littoral atlantique. Ailleurs, la surface libre profonde (jusqu'à $120 \mathrm{~m}$ ) et les faibles débits spécifiques en limitent l'intérêt économique.

\section{Applications de la géochimie isotopique} 2.1. Géochimie des eaux souterraines. Importance de la
perturbation de la teneur à l'origine en carbone 14 .

L'étude hydrogéochimique précise a un double but : correction des données sur l'activité du carbone 14, perturbées par la présence de carbonates dans la roche réservoir, et vérification des hypothèses géologiques et hydrodynamiques concernant l'alimentation naturelle, le renouvellement et l'écoulement des eaux souterraines. Les données isotopiques doivent toujours être intégrées dans leur contexte hydrogéochimique dont elles ne peuvent être dissociées.

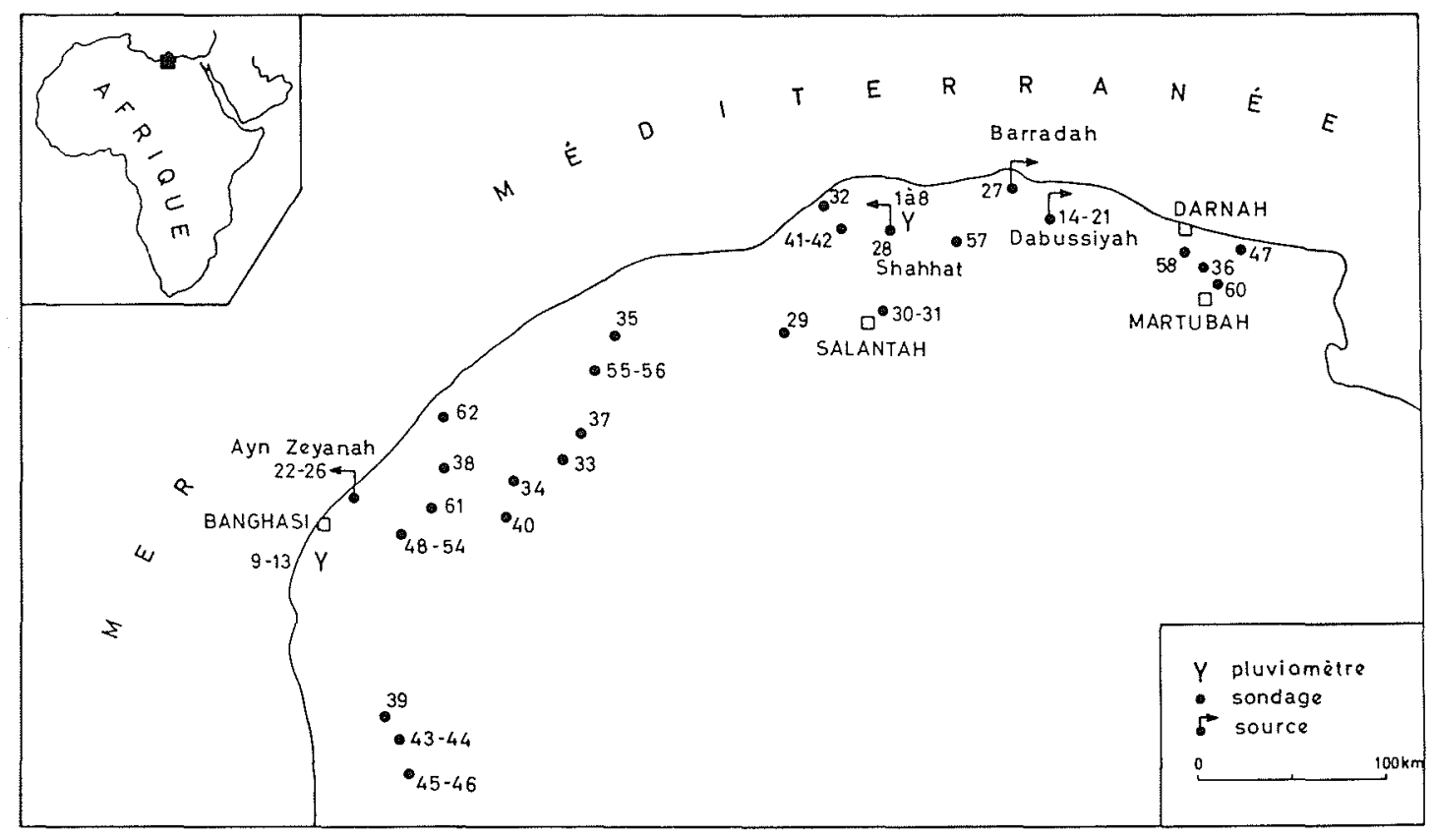

$2 /$ Nappe des calcaires miocènes de Cyrénaique (Lybie)

La zone hydrogéologique de Cyrénaïque étudiée, d'une surface de $14000 \mathrm{~km}^{2}$, correspond approximativement au massif littoral du Jebel Al-Akhdar (fig. 2). Elle est constituée par un anticlinal faillé découpé morphologiquement en trois plateaux qui s'étagent successivement de la côte vers le Sud, à des altitudes de 0-200, 256-300 et $450-500 \mathrm{~m}$. Le réservoir aquifère principal est constitué essentiellement par une puissante formation de calcaires miocènes karstifiés. Les eaux souterraines sont souvent captives. Les exutoires naturels sont d'importantes sources littorales : Ayn-Zayanah dans la région de Benghazi $\left(3 \mathrm{~m}^{3} / \mathrm{s}\right)$, Shahhat, Barradah, Dabusiyah dans le secteur oriental (fig. 2). De nombreux forages profonds captent des eaux souterraines. Les réserves totales sont estimées de 13 à $17.10^{9} \mathrm{~m}^{3}$.

Le bassin d'Aïn-Beni-Mathar (Maroc Oriental) comporte un aquifère dans des calcaires dolomitiques (avec gypse et anhydrite) fracturés du Jurassique inférieur et moyen, compartimentés par des failles. La nappe est en majeure partie captive. Les deux exutoires principaux sont les sources Rass-elAin $(500 \mathrm{l} / \mathrm{s})$ et Guefait $(150$ à $900 \mathrm{I} / \mathrm{s})$.

\section{Hydrogéochimie des carbonates et carbone 13}

Nous attirons l'attention sur la géochimie des carbonates, base indispensable de l'interprétation des résultats concernant les isotopes du carbone. Elle devient encore plus nécessaire en présence de réservoirs de roches carbonatées car des échanges entre la matrice poreuse et l'eau sont possibles; dans ce cas, intervient le contrôle indispensable, bien qu'imparfait, par le carbone 13, indicateur d'éventuels échanges isotopiques dont la conséquence est le vieillissement apparent de l'eau.

Par exemple, pour chaque échantillon d'eau de la nappe des Sables "maestrichtiens" du Sénégal, le rapport ${ }^{14} \mathrm{C} /{ }^{13} \mathrm{C}$ a été calculé. Certains résultats montrent des teneurs normales en carbone 13 décelant une probabilité d'échanges. L'écart avec la normale a servi de base pour corriger l'activité en ${ }^{14} \mathrm{C}$. Une carte des teneurs en carbone 13 (fig. 1.) montre des valeurs normales dans le secteur centre-Nord et en deux points au $S W$ et au $N E$. A la périphérie est et nord et dans toute la région méridionale, la teneur indique des échanges isotopiques avec la roche réservoir. 
De même, en Cyrénaïque, les teneurs en ${ }^{13} \mathrm{C}$ montrent des échanges entre les calcaires du réservoir et l'eau (quatre échantillons sont perturbés à $100 \%$ ).

Pour la nappe des calcaires dolomitiques d'Aïn-Beni-Mathar, si ces échanges isotopiques semblent négligeables (homogénéité des teneurs en ${ }^{13} \mathrm{C}$ ), un vieillissement apparent des "âges" par solubilité partielle des carbonates se manifeste.

Ces études ont été complétées pour la nappe des Sables "maestrichtiens" du Sénégal par deux cartes, l'une des teneurs en bicarbonates et l'autre du degré de saturation de l'eau par rapport à la calcite. Cette dernière fut réalisée pour tenir compte des perturbations éventuelles du rapport isotopique ${ }^{14} \mathrm{C} /{ }^{12} \mathrm{C}$ entrainées par dissolution des carbonates dont l'activité en ${ }^{14} \mathrm{C}$ peut être considérée comme nulle.

\section{Hydrogéochimie}

Des représentations synthétiques des données physicochimiques décrivant l'évolution hydrogéochimique globale sont utiles. Ce sont des cartes hydrogéochimiques (concentration totale, rapports caractéristiques, indice d'échanges de bases, isothermes) et des diagrammes représentatifs des éléments majeurs (diagrammes logarithmiques de SchoellerBerkaloff et trilinéaires de Piper).

La concentration totale (et la teneur en sulfates) des eaux de la nappe des Sables "maestrichtiens" du Sénégal augmente, d' $E$ en $W$, de 250 à $2000 \mathrm{mg} / \mathrm{l}$. L'étude géochimique montre d'une part que le remplissage de l'aquifère remonte à une période très ancienne, et d'autre part confirme l'existence de pertes à travers son toit. Le faciès géochimique est sulfaté sodique carbonaté avec enrichissement en chlorures vers le littoral. Cette évolution confirme un confinement de l'écoulement vers l'Ouest.

Les eaux des calcaires de Cyrénaïque sont bicarbonatées calcique évoluant vers le type bicarbonaté sodique. Vers le littoral, la concentration augmente avec un type chloruré calcique décelant une invasion des eaux marines.

Le faciès des eaux du bassin de l'Aïn-Beni-Mathar est sulfaté calcique bicarbonaté.

Les conditions requises pour les connaissances de base hydrogéologiques et hydrogéochimiques étant remplies, l'étude des isotopes du milieu apporte, outre un contrôle précieux des connaissances acquises, ou des hypothèses formulables, des données nouvelles pour la gestion des ressources en caux.

\subsection{Interprétation des données du carbone 14}

C'est surtout le carbone 14 qui est un bon traceur naturel du déplacement des eaux souterraines dans les aquifères de grandes dimensions. Nous citerons deux méthodes d'interprétation : les variations de la teneur en carbone 14 de leau avec sa profondeur de gisement et les cartes des "âges" apparents de l'eau souterraine.

Variation de la teneur en carbone 14 de l'eau avec sa profondeur de gisement

Globalement, dans les nappes étudiées, l'“âge" apparent de l'eau s'accroît avec la profondeur. Mais, dans le détail, des anomalies apparaissent dûes à l'hétérogénéité de la roche réservoir et à des échanges isotopiques avec les carbonates de la formation.

\section{Cartes de l"age" apparent de l'eau souterraine}

Des cartes des "âges" apparents (ou d'isoradioactivité du carbone 14) de l'eau souterraine sont dressćes. L' "âge" apparent est obtenu après correction des valeurs du rapport ${ }^{14} \mathrm{C} /{ }^{12} \mathrm{C}$ à partir de ${ }^{13} \mathrm{C} /{ }^{12} \mathrm{C}$. Ces documents permettent de dresser une synthèse des résultats, ne signifiant nullement que le processus d'alimentation est demeuré constant au cours de la période considérée, mais commode pour les interprétations spatio-temporelles. Avec une répartition favorable des points de mesure, ces cartes permettent de préciser les conditions d'alimentation des aquifères (localisation des zones d'alimentation actuelle et évolution de la recharge dans le temps) et les caractéristiques générales de l'écoulement de la nappe (direction et vitesse de déplacement).

Ainsi, pour la nappe des Sables "maestrichtiens" du Sénégal, cette cartographie (fig. 3) a confirmé l'existence d'une alimentation principale actuelle au $S E$ du système malgré un recouvrement irrégulier de terrains peu perméables et le long du fleuve Sénégal à l'E. Par contre, des apports récents, toutefois peu importants, ont été décellés au $N-N W$ alors que l'étude hydrogéologique concluait plutôt à une zone d'exutoire naturel.

Ces processus d'alimentation, étant donné la régularité du gradient d" "âge" apparent observé, n'ont pas été sensiblement modifiés pendant une durée importante (10000 à 20000 ans au moins). Deux phases dans l'évolution de l'alimentation dans le temps ont été mises en évidence, l'une principale régulière, et ancienne au $S E$, laquelle se poursuit actuellement, l'autre récente au $N$ et à l' $E$ en relation directe avec le fleuve Sénégal. Il semble que le secteur nord a été influencé au cours de la dernière transgression marine, cause probable de variations sensibles de niveau relatif mer/continent.

Pour la nappe du Continental terminal (fig. 4), la répartition des "âges" observée n'est pas celle déduite de la carte piézométrique actuelle. Un apport d'eau très récent au centre de la dépression piézométrique de la nappe du Ferlo ne se traduit pas par un dôme local de la surface libre, laquelle est au contraire très déprimée ("nappe en creux"). Cette dépression, ne correspondant donc pas à l'état actuel, résulterait de l'influence d'une période immédiatement antérieure au cours de laquelle le climat a pu etre beaucoup plus aride que l'actuel. Le reste de la nappe, soit la majorité de sa surface, est constitué d'une eau très ancienne.

A Aïn-Beni-Mathar, l'eau de la nappe provient des reliefs jurassiques du Haut et (ou) du Moyen Atlas. Bien que d'origine ancienne, elle a reçu des apports relativement récents en plusieurs points de l'aquifère.

Nous noterons l'intérêt de la confrontation de la carte piézométrique avec celle des "âges" apparents. Les vitesses de déplacement, déterminées par les données isotopiques, sont en général, après les corrections nécessaires, en bonne concordance avec les vitesses moyennes d'écoulement obtenues par les méthodes hydrodynamiques : 8 à $30 \mathrm{~m}$ par an pour la nappe des Sables "maestrichtiens" du Sénégal.

\subsection{Apports des teneurs en tritium}

Les données sur les teneurs en tritium permettent, dans le contexte précédent, un contrôle par l'étude de leur cohérence avec celles du carbone 14. Elles détectent l'apport ponctuel d'eaux récentes. Des mélanges d'eaux d' "âges" différents ont été mis en évidence dans la nappe de Cyrénaique (Salantah, 5450 pour ${ }^{14} \mathrm{C}$ et après 1958 pour le tri- 


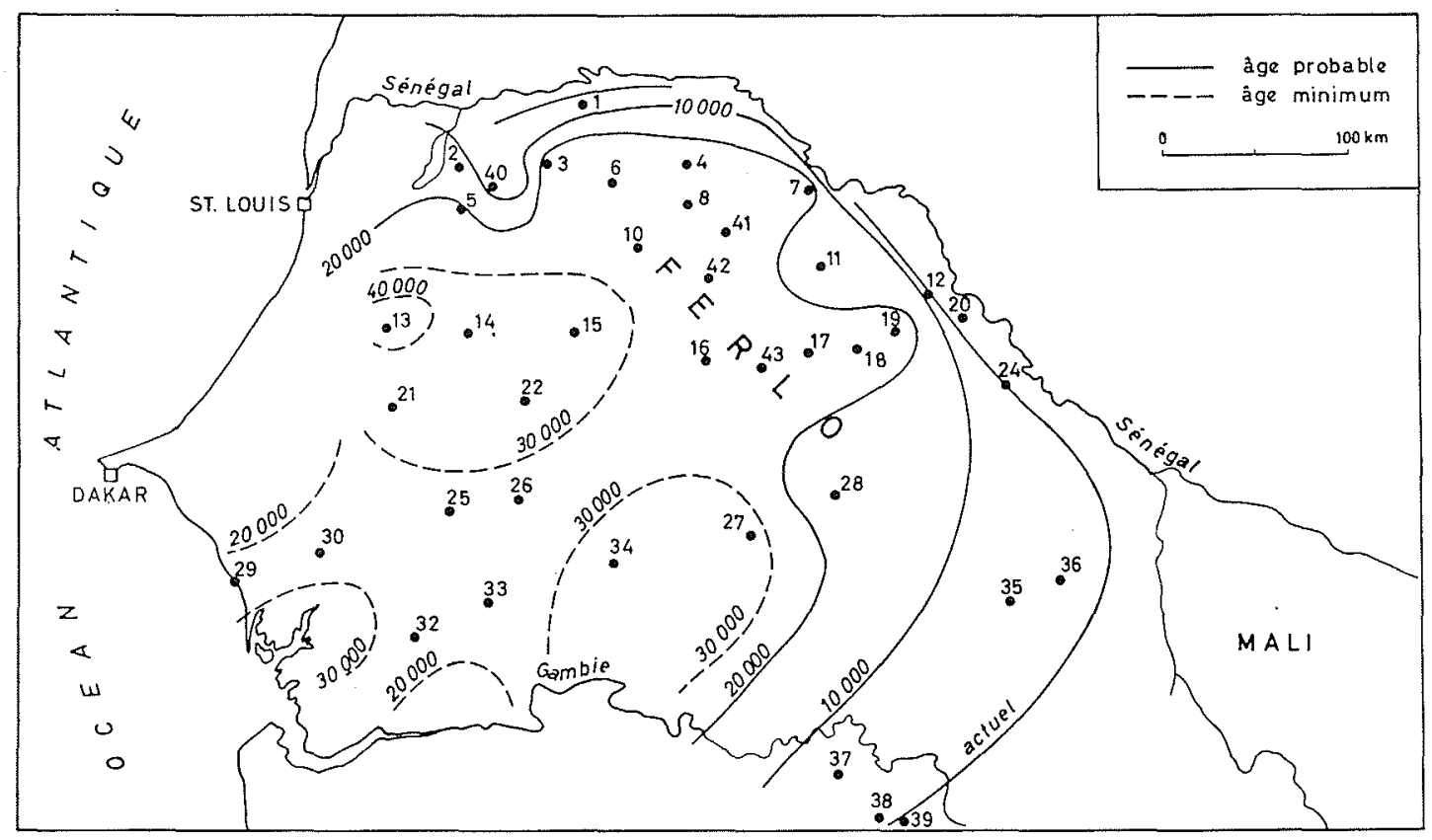

$3 /$ Nappe des sables "maestrichtiens" du Sénégal. Age apparent ${ }^{14} \mathrm{C}$

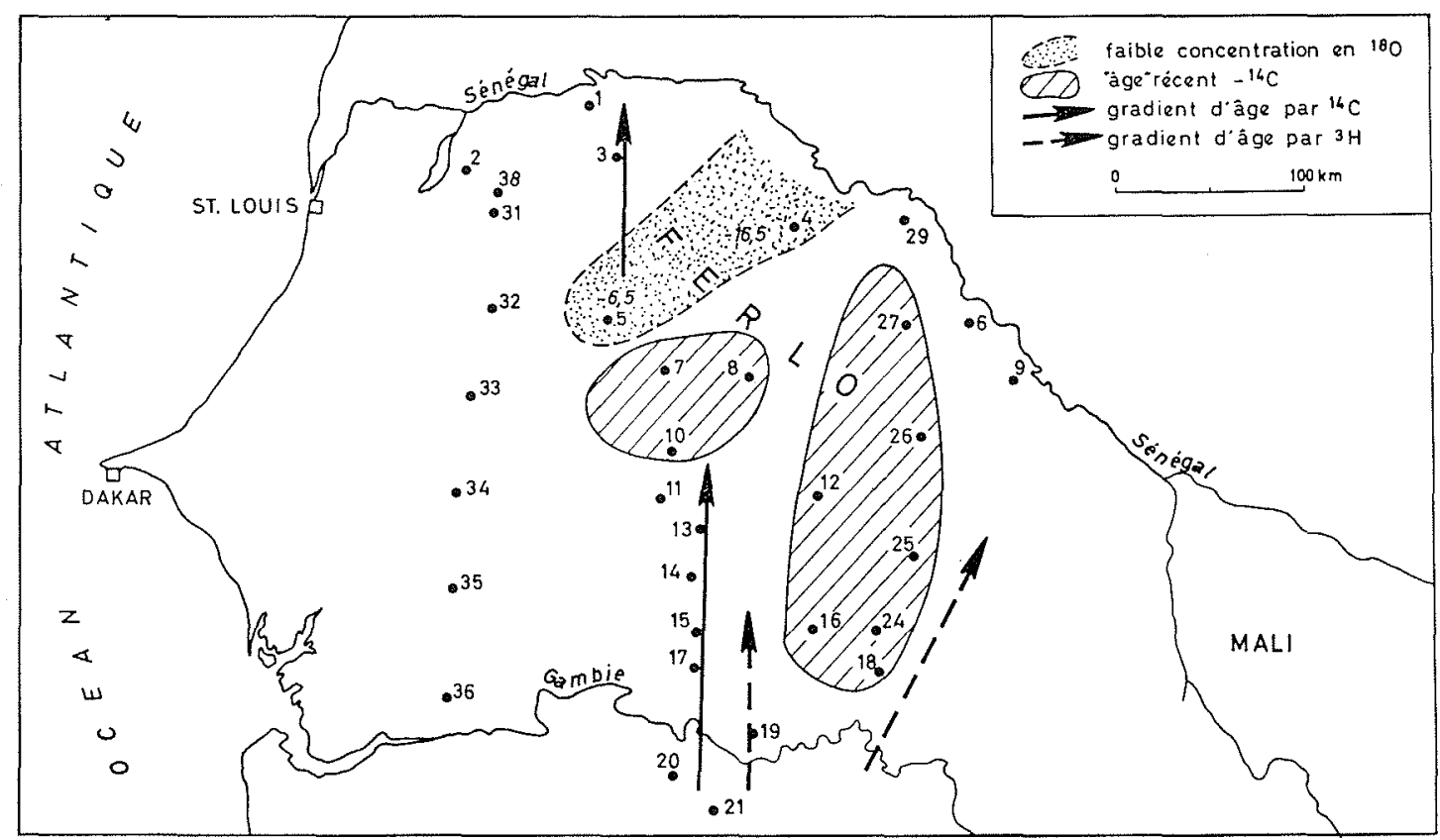

4/ Nappe du Continental terminal du Sénégal. Teneurs en ${ }^{14} \mathrm{C}$ et ${ }^{18} \mathrm{O}$

tium) et d'Aïn-Beni-Mathar. Ces observations entrainent d'importantes conclusions sur l'alimentation actuelle et le renouvellement des eaux souterraines.

\subsection{Apport des teneurs en oxygène 18}

Des données sur les teneurs en oxygène 18 ont été recueillies, tant sur les eaux de pluie que sur les eaux souterraines. Pour la nappe des Sables "maestrichtiens" du Sénégal, les valeurs de ${ }^{18} \mathrm{O}$, homogènes (normales de $-5,5$ à $-6,5 \%$ Smow) confirment celles du carbone 14. Leur moyenne de $-6 \%$ 士 $5 \%$ Smow étant sensiblement différente de celle interannuelle, calculée à Bamako $-4,8 \%$ Smow. L'eau de pluie actuelle est sensiblement différente de celle de la nappe. Par contre, dans la nappe du Continental terminal, deux groupes apparaissent, l'un autour des valeurs $-5,5 \%$, l'autre vers $-6,5 \%$ smow traduisant une hétérogénéité provoquée soit par la lithologie du réservoir, soit par les variations interannuelles des teneurs en ${ }^{18} \mathrm{O}$ de l'eau des précipitations mises en évidence à la station de Bamako.

Dans la nappe des calcaires miocènes de Cyréna ique, deux groupes s'individualisent. L'un avec Ayn-Zeyanah, à l'W, est marqué par une dilution de l'eau souterraine par celle des précipitations (composition isotopique en ${ }^{18} \mathrm{O}$ correspondant à une altitude et des températures analogues à celles de Benghazi), l'autre, localisé à la zone de Shabbat, correspondant à 
des conditions thermodynamiques analogues. Dans la nappe d'Ain-Beni-Mathar, les teneurs en ${ }^{18} \mathrm{O}(-8,5 \pm 0,1 \%$ à $8,2 \pm$ $0,1 \%$ Smow) marquent une bonne corrélation avec celles du carbone 14. Elles confirment une alimentation provenant de reliefs nettement plus élevés $(1.800$ à $2.500 \mathrm{~m}$ ) que celle du gisement actuel des eaux souterraines, sans qu'il soit possible de préciser si cet apport s'effectue par le Haut Atlas et (ou) le Moyen Atlas.

\section{Conclusions}

Les exemples précédents illustrent assez bien les types de contribution apportée par les "techniques isotopiques" à la connaissance des systèmes aquifères.

En premier lieu, ces méthodes concourent à la schématisation de la structure interne des réservoirs aquifères.

Dans certaines conditions, la géochimie isotopique permet le contrôle de l'homogénéité, ou de l'hétérogénéité du réservoir par l'étude de la répartition des principaux isotopes (carbone 14 et oxygène 18). Si la nappe des Sables "maestrichtiens" du Sénégal est homogène, par contre celle du Continental terminal montre une certaine hétérogénéité, laquelle avait d'ailleurs été décelée par l'hydrogéochimie. A Ainn-Beni-Mathar, la répartition composite dans la distribution des "âges" apparents montre une structure profonde très compartimentée, résultat de nombreuses fractures mises en évidence par les études de géologie structurale.

En second lieu, ces méthodes concourent à la mise en évidence des conditions d'alimentation des aquifères, notamment de leur degré d'actualité et de la localisation des apports, informations primordiales pour définir correctement les conditions aux limites. Ces informations sont d'autant plus utiles que les données piézométriques sont souvent insuffisantes au voisinage des limites de certains grands aquifères sédimentaires.

En sus des cas exposés ici, d'autres études récentes ont présenté des exemples d'application probants, notamment pour préciser les conditions d'alimentation de nappes libres de grande étendue en zone semi-aride.

Citons l'étude de la vallée du Souss au Maroc par l'A.I.E.A. (Payne et Sauzay 1974 [7].

Des informations peuvent encore être obtenues sur l'importance réelle des transferts d'eau par drainance dans les systèmes aquifères multicouches.

On sait qu'une des caractéristiques importantes de la dynamique des eaux souterraines dans les grands bassins sédimentaires est la drainance, laquelle peut provoquer, à travers les intercalations semi-perméables, des transferts d'eau souterraine significatifs, Les conditions d'alimentation et de décharge d'une nappe déterminée sont ainsi modifiées. Lorsqu'ils sont appréciables, ces apports peuvent être mis en évidence par les isotopes du milieu. Ainsi, la carte des "âges" apparents de la nappe des Sables "maestrichtiens" du Sénégal a permis de confirmer l'existence de transferts d'eau par drainance avec l'aquifère superposé du Continental terminal : alimentation au sud et pertes au nord de la Gambie. Au Maroc oriental, des phénomènes analogues sont reconnus. Les affleurements des formations du Jurassique moyen de la chaîne des Trarit, superposés à l'aquifère, constituent actuellement des zones d'alimentation appréciables. Ce fait infirme l'une des hypothèses de l'étude hydrogéologique. Toutefois, cet apport ne représente qu'un faible renouvellement des réserves de l'aquifère. Ces faits montrent l'unité hydrologique de deux aquifères superposés, séparés par une assise semi-perméable (aquifère bicouche ou multicouche).

En conclusion, on peut affirmer que, si les données d'analyse isotopique n'apportent pas par elles-mêmes d'information globale sur les ressources en eau souterraine, hors du contexte hydrogéologique structural et hydrodynamique (quelques illusions sur la possibilité d'une "voie directe" avaient été parfois entretenues. . .), en revanche, elles peuvent contribuer très efficacement et parfois de manière irremplaçable, intégrées avec d'autres informations, à définir la structure et le comportement dynamique des systèmes aquifères dont la connaissance est indispensable pour évaluer les ressources exploitables.

\section{Références}

[1] Audibert (M.) - Etude hydrogéologique de la nappe profonde du Sénégal, "nappe maestrichtienne" - Mém. B.R.G.M. 411966.

(2) DEPAGNE (J.) , Moussu (H.) - Carte hydrogéologique de la République du Sénégal, à $1 / 500000$ - Direction de l'Hydraulique, Dakar. 1967.

[3] Depagne (J.), Martin (A.), Moussu (H.), Napias (JC.) Carte hydro-chimique des nappes phréatiques de la République du Sénégal à $1 / 1000$ 000. Direction de I'Hydraulique, Dakar. 1966.

[4] EVIN (J.), Vuillaume (Y.) - Etude par le radiocarbone de la nappe captive de l'Albien du Bassin de Paris "Isotope hydrology symposium", Vienne, AIEA SM 129-19. 1970.

[5] VuILlaume (Y.) - Application des méthodes isotopiques et hydrochimiques à l'étude de la nappe de l'Albien dans le Bassin de Paris. Thèse, Paris. 1971

[6] Castany (G.), Evin (J.), Marce (A.), Margat (J), Moussu (H.), VuillaUmE (Y.) - Etude par les isotopes du milieu du régime des eaux souterraines dans les aquifères de grandes dimensions. (A.I.E.A., Isotopes techniques in groundwater hydrology, I, p. 249-258, Vienne). 1974

[7] PAYNE (B.R.), SAUZAY (G.) - Contribution des traceurs isotopiques naturels à l'étude de l'alimentation de la nappe phréatique de l'Oued Souss (Maroc) - (Bull. B.R.G.M. Sec. III, n 3, p. 227-243, Paris). 1974 


\section{Discussion}

Président : M. Jean MARGAT

M. Le Président remercie M. MARCE de son exposé et ouvre la discussion.

Il n'est pas possible, observe M. Guizerix, de parler d'un "äge" sur la base d'un échantillon. Pour la clarté de l'exposé, il serait bon que l'influence de la distribution des temps de séjour soit soulignée.

Il n'est pas possible, en effet, répond M. MARCE de délivrer un "certificat de naissance" pour telle ou telle eau souterraine mais dans l'état actuel de nos connaissances, il est parfois nécessaire et suffisant de distinguer un apport d'eau récent d'un apport ancien de façon à pouvoir se rendre compte de l'existence, aujourd'hui, d'une recharge notable de la nappe.

Dans certaines régions -du Sahara notamment - où l'examen du bilan de la nappe concluait à une absence de recharge, la détection de concentrations mesurables de tritium ou de carbone 14 a montré que la recharge de la nappe était une réalité (1)

M. DEGALLIER conteste le fait que les radioisotopes aient permis de déceler, pour la première fois, la réalimentation (ou la non réalimentation de telle ou telle nappe) ; les méthodes plus anciennes y avaient suffi. Pour lui, les méthodes radioisotopiques présentent un intérêt pour les recherches d'avant-garde mais n'ont pas encore de notables applications pratiques.

(1) Mise en évidence, par le tritium, de la recharge actuelle des nappes libres de la zone aride saharienne (ALGERIE) par G. CONRAD, A. MARCE, Ph. OLIVE. Journal of Hydrology 27 (1975), 207-224.
D'ailleurs, poursuit M. DEGALLIER, on observe qu'en dehors de quelques points particuliers --.toujours assez isolés-- où les teneurs en carbone 14 indiquent des ages faibles, partout ailleurs -dans les grands bassins d'Afrique ou d'Asie comme dans ceux des Etats-Unis- on aboutit à des âges compris entre 20000 et 50000 ans, c'est-à-dire à des valeurs excédant les limites de précision de la méthode.

M. Paul Ch. LEVEQUE intervient en ces termes :

"Je ne suis pas entièrement d'accord avec l'intervention de $M$. DEGALLIER qui estime l'utilisation du tritium, du carbone 14 et de l'oxygène 18 , limitée au domaine des recherches théoriques.

"Ces trois isotopes sont utilisés par des ingénieurs dans des travaux hydrauliques dont le caractère évident est l'obligation de serrer de près la réalité. Nous n'avons pas le temps de citer d'exemples, mais nous supposons que les trois ou quatre organismes français qui travaillent dans cette direction, ont, dans leurs cartons, des cas dapplication typiques".

L'évaluation des ressources en eau souterraines, remarque $M$. Le Président, n'est pas le principal champ d'application de ce qu'on appelle la "technique isotopique" ni surtout celui où son utilisation est la plus efficace.

Par contre, il y a bien d'autres problèmes auxquels les ingénieurs ont à faire face -et là, je rejoins le Professeur Paul Ch. LEvEQuE- où les mesures isotopiques, qu'il s'agisse d'isotopes naturels ou de traceurs artificiels, trouvent un très large domaine d'emploi.

M. Le Président clôt ainsi la discussion et donne la parole à $\mathrm{M}$. MANGIN pour l'exposé de la communication qu'il a établie en collaboration avec MM. PA LOC et M. MOLINARI. 